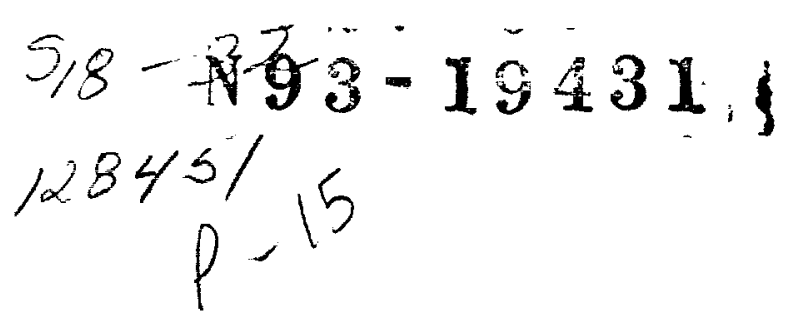

\title{
Multiple Symbol Partially Coherent Detection of MPSK
}

\author{
M. K. Simon \\ Telecommunications Systems Section \\ D. Divsalar \\ Communications Systems Research Section
}

\begin{abstract}
In this article, it is shown that by using the known (or estimated) value of carrier tracking loop SNR in the decision metric, it is possible to improve the error probability performance of a partially coherent multiple phase-shift-keying (MPSK) system relative to that corresponding to the commonly used ideal coherent decision rule. Using a maximum-likelihood approach, an optimum decision metric is derived and shown to take the form of a weighted sum of the ideal coherent decision metric (i.e., correlation) and the noncoherent decision metric which is optimum for differential detection of MPSK. The performance of a receiver based on this optimum decision rule is derived and shown to provide continued improvement with increasing length of observation interval (data symbol sequence length). Unfortunately, increasing the observation length does not eliminate the error floor associated with the finite loop SNR. Nevertheless, in the limit of infinite observation length, the average error probability performance approaches the algebraic sum of the error floor and the performance of ideal coherent detection, i.e., at any error probability above the error floor, there is no degradation due to the partial coherence. It is shown that this limiting behavior is virtually achievable with practical size observation lengths. Furthermore, the performance is quite insensitive to mismatch between the estimate of loop SNR (e.g., obtained from measurement) fed to the decision metric and its true value. These results may be of use in low-cast Earth-orbiting or deep-space missions employing coded modulations.
\end{abstract}

\section{Introduction}

It is well known that for ideal phase coherent detection of multiple phase-shift-keying (MPSK), the decision rule that minimizes average bit error probability is based on a correlation metric and leads to bit-by-bit decisions. In practical situations, the phase introduced by the transmission over the channel is unknown and thus the assumption of perfect knowledge of this parameter at the receiver is idealistic. Typically, if the channel phase is reasonably well behaved, the receiver will attempt to estimate it via some type of phase synchronization subsystem, such as a carrier phase tracking loop. Since the estimate is made in the presence of the ever-present additive channel thermal noise, the receiver's phase estimate used for demodulation purposes is not perfect. Detection under these circumstances is known as partially coherent detection.

Ordinarily in this environment, one continues to use the ideal coherent detection correlation metric despite the fact that it is no longer optimum for partially coherent detec- 
tion. In particular, the presence of a phase error between the true channel and the receiver's estimate of it introduces memory into the observation, and thus any metric leading to bit-by-bit detection cannot be optimum. Instead, one must resort to sequence estimation where the length of the sequence is proportional to the duration over which the phase error can be assumed constant.

In this article, a maximum-likelihood approach to partially coherent detection is taken, an approach not unlike that previously applied to noncoherent and coherent detection. It will be shown that considerable performance improvement can be gained by using the optimum metric which leads to a maximum-likelihood sequence estimation (MLSE) type of algorithm.

\section{Maximum-Likelihood Partially Coherent Detection of MPSK Over an AWGN Channel}

Consider the transmission of MPSK signals over an additive white Gaussian noise (AWGN) channel. The baseband representation of the transmitted signal in the interval $(k T,(k+1) T)$ has the complex form

$$
s_{k}=\sqrt{2 P} e^{j \phi_{k}}
$$

where $P$ denotes the constant signal power, $T$ denotes the MPSK symbol interval, and $\phi_{k}$ the transmitted phase which takes on one of $M$ uniformly distributed values $\beta_{m}=2 \pi m / M ; m=0,1, \ldots, M-1$ around the unit circle. Assume that in addition to AWGN, the channel introduces a phase $\theta$ which can be constant (independent of time) over a duration of $N$ data symbols and uniformly distributed in the interval $(-\pi, \pi)$. Thus, the received sequence $\mathbf{r}$ is expressed as

$$
\mathbf{r}=\mathbf{s} e^{j \theta}+\mathbf{n}
$$

where $\mathbf{r}=\left(r_{0}, r_{1}, \ldots, r_{N-1}\right), \mathbf{s}=\left(s_{0}, s_{1}, \ldots, s_{N-1}\right)$, and $\mathbf{n}=\left(n_{0}, n_{1}, \ldots, n_{N-1}\right)$ are the received sequence, transmitted sequence, and noise sequence, respectively. Also, $n_{k}$ is a sample of zero mean complex Gaussian noise with variance (per dimension) $\sigma_{n}^{2}=N_{0} / T$ where $N_{0}$ is the singlesided power spectral density of the noise process $n(t)$ at the receiver input.

For partially coherent detection, the receiver provides a carrier phase synchronization subsystem, e.g., a tracking loop, which derives a complex reference signal $e^{j \hat{\theta}}$ whose phase $\hat{\theta}$ is an estimate of the unknown channel phase $\theta$. After demodulating $\mathbf{r}$ with this reference (complex conjugate multiplication of the two signals), one gets

$$
\mathbf{R}=\mathbf{r} e^{-j \hat{\theta}}=\mathbf{s} e^{j \phi_{c}}+\mathbf{n} e^{-j \hat{\theta}}
$$

where $\phi_{c} \triangleq \theta-\hat{\theta}$ is the carrier phase error and typically has a Tikhonov probability density function (pdf) [1], i.e.,

$$
p\left(\phi_{c}\right)=\frac{\exp \left(\rho \cos \phi_{c}\right)}{2 \pi I_{0}(\rho)} ; \quad\left|\phi_{c}\right| \leq \pi
$$

Here $\rho$ is a parameter related ${ }^{1}$ to the tracking loop SNR and $I_{0}($.$) is the zeroth-order modified Bessel function of$ the first kind.

For the assumed AWGN model for $\mathbf{n}$, the a posteriori probability of the demodulated received sequence $\mathbf{R}$ given the transmitted sequence $s$ and the carrier phase error $\phi_{c}$ follows from Eq. (3) and is

\footnotetext{
${ }^{1}$ For first-order tracking loops, $\rho$ is indeed the loop SNR. For secondorder loops, $\rho$ is approximately the loop SNR for sufficiently large values [1]. In what follows, $\rho$ is referred to simply as the loop SNR which is assumed to be known or estimated.
}

$$
\begin{aligned}
p\left(\mathbf{R} \mid \mathbf{s}, \phi_{c}\right) & =\frac{1}{\left(2 \pi \sigma_{n}^{2}\right)^{N}} \exp \left\{-\frac{\left\|\mathbf{R}-\mathbf{s} e^{j \phi_{c}}\right\|^{2}}{2 \sigma_{n}^{2}}\right\} \\
& =\frac{1}{\left(2 \pi \sigma_{n}^{2}\right)^{N}} \exp \left\{-\frac{1}{2 \sigma_{n}^{2}}\left[\sum_{i=0}^{N-1}\left[\left|R_{k-i}\right|^{2}+\left|s_{k-i}\right|^{2}\right]-2\left|\sum_{i=0}^{N-1} R_{k-i} s_{k-i}^{*}\right| \cos \left(\phi_{c}-\alpha\right)\right]\right\}
\end{aligned}
$$


where

$$
\alpha=\tan ^{-1} \frac{\operatorname{Im}\left\{\sum_{i=0}^{N-1} R_{k-i} s_{k-i}^{*}\right\}}{\operatorname{Re}\left\{\sum_{i=0}^{N-1} R_{k-i} s_{k-i}^{*}\right\}}
$$

Averaging Eq. (5) over the pdf in Eq. (4) gives, upon simplification,

$$
\begin{aligned}
p(\mathbf{R} \mid \mathbf{s})= & \int_{-\pi}^{\pi} p\left(\mathbf{R} \mid \mathbf{s}, \phi_{c}\right) p\left(\phi_{c}\right) d \phi_{c} \\
= & \frac{1}{I_{0}(\rho)} \frac{1}{\left(2 \pi \sigma_{n}^{2}\right)^{N}} \exp \left\{-\frac{1}{2 \sigma_{n}^{2}} \sum_{i=0}^{N-1}\left[\left|R_{k-i}\right|^{2}+\left|s_{k-i}\right|^{2}\right]\right\} \\
& \times I_{0}\left(\frac{1}{\sigma_{n}^{2}} \sqrt{\left(\left|\sum_{i=0}^{N-1} R_{k-i} s_{k-i}^{*}\right| \cos \alpha+\rho \sigma_{n}^{2}\right)^{2}+\left(\left|\sum_{i=0}^{N-1} R_{k-i} s_{k-i}^{*}\right| \sin \alpha\right)^{2}}\right)
\end{aligned}
$$

Since

$$
\left|\sum_{i=0}^{N-1} R_{k-i} s_{k-i}^{*}\right| \cos \alpha=\operatorname{Re}\left\{\sum_{i=0}^{N-1} R_{k-i} s_{k-i}^{*}\right\} ;\left|\sum_{i=0}^{N-1} R_{k-i} s_{k-i}^{*}\right| \sin \alpha=\operatorname{Im}\left\{\sum_{i=0}^{N-1} R_{k-i} s_{k-i}^{*}\right\}
$$

Eq. (7) further simplifies to

$$
\begin{aligned}
p(\mathbf{R} \mid \mathbf{s})= & \frac{1}{I_{0}(\rho)} \frac{1}{\left(2 \pi \sigma_{n}^{2}\right)^{N}} \exp \left\{-\frac{1}{2 \sigma_{n}^{2}} \sum_{i=0}^{N-1}\left[\left|R_{k-i}\right|^{2}+\left|s_{k-i}\right|^{2}\right]\right\} \\
& \times I_{0}\left(\frac{1}{\sigma_{n}^{2}} \sqrt{\left(\operatorname{Re}\left\{\sum_{i=0}^{N-1} R_{k-i} s_{k-i}^{*}\right\}+\rho \sigma_{n}^{2}\right)^{2}+\left(\operatorname{Im}\left\{\sum_{i=0}^{N-1} R_{k-i} s_{k-i}^{*}\right\}\right)^{2}}\right)
\end{aligned}
$$

Note from Eq. (1) that for MPSK, $\left|s_{k}\right|^{2}$ is constant for all transmitted phases $\beta_{m}$. Thus, since $I_{0}(x)$ is a monotonic function of its argument, maximizing $p(\mathbf{R} \mid \mathbf{s})$ over $\mathbf{s}$ is equivalent to finding

$$
\begin{aligned}
\max _{s}\left\{\left(\operatorname{Re}\left\{\sum_{i=0}^{N-1} R_{k-i} s_{k-i}^{*}\right\}+\rho \sigma_{n}^{2}\right)^{2}+\left(\operatorname{Im}\left\{\sum_{i=0}^{N-1} R_{k-i} s_{k-i}^{*}\right\}\right)^{2}\right\}= \\
\max _{s}\left\{\left|\sum_{i=0}^{N-1} R_{k-i} s_{k-i}^{*}+\rho \sigma_{n}^{2}\right|^{2}\right\}=\max _{s}\left\{\left|\sum_{i=0}^{N-1} R_{k-i} s_{k-i}^{*}\right|^{2}+2 \rho \sigma_{n}^{2} \operatorname{Re}\left\{\sum_{i=0}^{N-1} R_{k-i} s_{k-i}^{*}\right\}\right\}
\end{aligned}
$$


This, using Eq. (1), results in the decision rule

$$
\text { choose } \hat{\phi}_{k}, \hat{\phi}_{k-1}, \ldots, \hat{\phi}_{k-N+1} \text { if }\left\{\left|\sum_{i=0}^{N-1} R_{k-i} \mathrm{e}^{-j \hat{\phi}_{n-i}}\right|^{2}+\frac{2 \rho \sigma_{n}^{2}}{\sqrt{2 P}} \operatorname{Re}\left\{\sum_{i=0}^{N-1} R_{k-i} \mathrm{e}^{-j \hat{\phi}_{k-i}}\right\}\right\} \text { is maximum }
$$

where $\hat{\phi}_{k}, \hat{\phi}_{k-1}, \ldots, \hat{\phi}_{k-N+1}$ is a particular sequence of the transmitted phases $\beta_{m}$. In Eq. (11), the first term inside the braces represents the component of the decision metric associated with noncoherent (differential) detection [2], i.e., total lack of knowledge of the uniformly distributed channel phase $\theta$. The second term inside the braces represents the component of the decision metric for ideal coherent detection, i.e., complete knowledge of the channel phase $\theta$. Thus, the partially coherent decision metric is a linear combination of the coherent and noncoherent decision metrics with the weighting of the two terms in proportion to the product of the tracking loop SNR and the channel noise variance. Note that for any nonzero value of $\rho$, this decision rule is unique because the second term inside the braces in Eq. (11) is unique but not the first term. For $\rho=0$, which corresponds to differentially coherent detection, there is a phase ambiguity since the addition of an arbitrary fixed phase, say $\phi_{a}$, to all $N$ estimated phases $\hat{\phi}_{k}, \hat{\phi}_{k-1}, \ldots, \hat{\phi}_{k-N+1}$ results in the same decision for $\phi$. In [2], the authors observed that by letting $\phi_{a}=\phi_{k-N+1}$ and differentially encoding the input phases at the transmitter,

$$
\phi_{k}=\phi_{k-1}+\Delta \phi_{k}
$$

where now $\Delta \phi_{k}$ denotes the input data phase corresponding to the $k$ th transmission interval and $\phi_{k}$ the differentially encoded version of it, the decision rule can turn into one in which the phase ambiguity is resolved. From now on, assume $\rho \neq 0$ and thus that there is no formal requirement for differentially encoding the data phase symbols.

Figure 1 is an illustration in complex form of a receiver implemented on the basis of Eq. (11). Note that this receiver requires knowledge of the loop SNR $\rho$, the signal power $P$, and the noise variance $\sigma_{n}^{2}$. The accuracy of this knowledge, which must be obtained by measurement, will have an impact on the ultimate performance of this receiver. Later, in Subsection E, the authors investigate the sensitivity of the receiver to a mismatch between the true loop SNR and the value supplied to the receiver implementation in Fig. 1. In the next section, except in Section III.E, it is assumed that the receiver has perfect knowledge of $\rho$, and thus should outperform a conventional bit-by-bit correlation receiver which does not make use of this knowledge. The following sections determine how much the optimum partially coherent sequence receiver outperforms the conventional bit-by-bit correlation receiver.

\section{Bit Error Probability Performance}

To obtain a simple upper bound on the average bit error probability, $P_{b}$, of the proposed $N$-bit detection scheme, use a union bound analogous to that used for upper bounding the performance of error correction coded systems. In particular, the upper bound on $P_{b}$ is the sum of the pairwise error probabilities associated with each $N$-bit error sequence. Each pairwise error probability is then either evaluated directly or itself upper bounded. Mathematically speaking, let $\phi=\left(\phi_{k}, \phi_{k-1}, \ldots, \phi_{k-N+1}\right)$ denote the sequence of $N$ transmitted information phases and $\hat{\phi}=\left(\hat{\phi}_{k}, \hat{\phi}_{k-1}, \ldots, \hat{\phi}_{k-N+1}\right)$ be the corresponding sequence of detected phases. Let $\mathbf{u}$ be the sequence of $b=N \log _{2} M$ information bits that produces $\phi$ at the transmitter and let $u$ be the sequence of $b$ bits that results from the detection of $\widehat{\phi}$ Then, since MPSK is a symmetric signalling set, i.e., it satisfies a uniform error probability (UEP) criterion, one gets an upper bound on the bit error probability,

$$
P_{b}\left(\phi_{c}\right) \leq \frac{1}{N \log _{2} M} \sum_{\hat{\phi} \neq \phi} w(\mathbf{u}, \hat{\mathbf{u}}) \operatorname{Pr}\left\{\hat{\eta}>\eta \mid \phi, \phi_{c}\right\}
$$

where the decision statistic $\eta$ is defined from Eqs. (10) and (11) by ${ }^{2}$

$$
\eta=\left|\sum_{i=0}^{N-1} R_{k-i} e^{-j \phi_{n-i}}+\frac{\rho \sigma_{n}^{2}}{\sqrt{2 P}}\right|^{2}
$$

\footnotetext{
2 Note that when compared with Eq. (11), $\eta$ of Eq. (14) includes the additional constant $\left(\rho \sigma_{n}^{2} / \sqrt{2 \bar{P}}\right)^{2}$. This, however, has no effect on the decision-making process and thus one can use the convenient form of Eq. (14) with no loss in generality.
} 
and the corresponding error statistic $\hat{\eta}$ is identical to Eq. (14) with each $\phi_{k}$ replaced by $\hat{\phi}_{k}$. In Eq. (13), w(u, û) denotes the Hamming distance between $\mathbf{u}$ and $\hat{\mathbf{u}}, \boldsymbol{\phi}$ is any input sequence (e.g., the null sequence $(0,0, \ldots, 0)=$ $0)$, and $\operatorname{Pr}\left\{\hat{\eta}>\eta \mid \phi, \phi_{c}\right\}$ denotes the pairwise probabil- ity that $\widehat{\phi}$ is incorrectly chosen when indeed $\phi$ was sent. Note that the bound in Eq. (13) is computed for a fixed carrier phase error, $\phi_{c}$, which accounts for the notational dependence of $\operatorname{Pr}\left\{\hat{\eta}>\eta \mid \phi, \phi_{c}\right\}$ and thus $P_{b}\left(\phi_{c}\right)$ on $\phi_{c}$.

\section{A. Evaluation of the Pairwise Error Probability}

To compute $\operatorname{Pr}\left\{\hat{\eta}>\eta \mid \phi, \phi_{c}\right\}$, the approach taken in [2] is used for evaluating the performance of multiple symbol differentially coherent detection of MPSK. In particular, letting $\eta=\left|z_{1}\right|^{2}$ and $\hat{\eta}=\left|z_{2}\right|^{2}$ [see Eq. (14) and the statement below it for the definitions of $z_{1}$ and $z_{2}$ ], then [3]

$$
\operatorname{Pr}\left\{\hat{\eta}>\eta \mid \phi, \phi_{c}\right\}=\frac{1}{2}[1-Q(\sqrt{b}, \sqrt{a})+Q(\sqrt{a}, \sqrt{b})] \triangleq f(a, b)
$$

where $Q(x, y)$ is the Marcum $Q$ function [4] and

$$
\left\{\begin{array}{l}
b \\
a
\end{array}\right\}=\frac{1}{2 N_{2}}\left\{\frac{S_{1}+S_{2}-2|\xi| \sqrt{S_{1} S_{2}} \cos \left(\theta_{1}-\theta_{2}+\nu\right)}{1-|\xi|^{2}} \pm \frac{S_{1}-S_{2}}{\sqrt{1-|\xi|^{2}}}\right\}
$$

where the + sign and - sign correspond to $b$ and $a$, respectively, and

$$
\begin{aligned}
& S_{1}=P\left|N+\frac{\rho}{2 E_{s} / N_{0}} e^{-j \phi_{c}}\right|^{2}=P\left(N^{2}+\frac{\rho}{E_{s} / N_{0}} N \cos \phi_{c}+\left(\frac{\rho}{2 E_{s} / N_{0}}\right)^{2}\right) \\
& S_{2}=P\left|\delta+\frac{\rho}{2 E_{s} / N_{0}} e^{-j \phi_{c}}\right|^{2}=P\left(|\delta|^{2}+\frac{\rho}{E_{s} / N_{0}} \operatorname{Re}\left\{\delta e^{j \phi_{c}}\right\}+\left(\frac{\rho}{2 E_{s} / N_{0}}\right)^{2}\right) \\
& N_{z}=\frac{1}{2}\left|\overline{z_{1}-\overline{z_{1}} \mid}\right|^{2}=N \frac{N_{0}}{T} \\
& \xi=\frac{1}{2 N_{z}} \overline{\left(z_{1}-\overline{z_{1}}\right)\left(z_{2}-\overline{z_{2}}\right)^{*}}=\frac{\delta}{N} ; \quad \nu=\arg \xi=\arg \delta \\
& \theta_{1}=\arg \overline{z_{1}}=\arg \left\{N e^{j \phi_{c}}+\frac{\rho}{2 E_{s} / N_{0}}\right\} ; \quad \theta_{2}=\arg \overline{z_{2}}=\arg \left\{\delta e^{j \phi_{0}}+\frac{\rho}{2 E_{o} / N_{0}}\right\}
\end{aligned}
$$

and

$$
\delta=\sum_{i=0}^{N-1} e^{j\left(\phi_{k-i}-\phi_{k-i}\right)_{0}}
$$

which is a normalized time cross correlation between $\mathbf{s}$ and $\hat{\mathbf{s}}$. Also, $E_{0} / N_{0} \triangleq P T / N_{0}$ denotes the symbol energy-to-noise spectral density ratio and is related to the bit energy-to-noise spectral density ratio $E_{b} / N_{0}$ by $E_{s} / N_{0}=\left(E_{b} / N_{0}\right) \log _{2} M$. Substituting Eq. (17) into Eq. (16) results, after considerable simplification, in 


$$
\begin{aligned}
\left\{\begin{array}{l}
b \\
a
\end{array}\right\}= & \frac{E_{s}}{2 N_{0}}\left\{N\left[1+\frac{1}{N}\left(\frac{\rho}{E_{s} / N_{0}}\right) \cos \phi_{c}+\frac{1}{2 N\left(N^{2}-|\delta|^{2}\right)}\left(\frac{\rho}{E_{s} / N_{0}}\right)^{2}(N-|\delta| \cos \nu)\right]\right\} \\
& \pm \frac{E_{s}}{2 N_{0}}\left[\sqrt{N^{2}-|\delta|^{2}}+\frac{1}{\sqrt{N^{2}-|\delta|^{2}}}\left(\frac{\rho}{E_{s} / N_{0}}\right)\left(N \cos \phi_{c}-|\delta| \cos \left(\phi_{c}+\nu\right)\right)\right]
\end{aligned}
$$

Now some special cases of practical interest are considered.

\section{B. Case 1: Binary PSK With Two-Symbol Observation and Detection ( $M=2, N=2)$}

In this case, $E_{s} / N_{0}=E_{b} / N_{0}$. There are $M^{2}-1=3$ possible error sequences each of length 2 . The pertinent results related to the evaluation of Eqs. (18) and (19) are

\begin{tabular}{ccr}
\hline$\phi_{k}-\hat{\phi}_{k}$ & $\phi_{k-1}-\hat{\phi}_{k-1}$ & \multicolumn{1}{c}{$\delta$} \\
\hline 0 & $\pi$ & 0 \\
$\pi$ & 0 & 0 \\
$\pi$ & $\pi$ & -2 \\
\hline
\end{tabular}

For the first two error sequences, Eq. (19) evaluates to

$$
\begin{aligned}
& b=\frac{E_{b}}{2 N_{0}}\left[4+2\left(\frac{\rho}{E_{b} / N_{0}}\right) \cos \phi_{c}+\frac{1}{4}\left(\frac{\rho}{E_{b} / N_{0}}\right)^{2}\right] \\
& a=\frac{E_{b}}{2 N_{0}}\left[\frac{1}{4}\left(\frac{\rho}{E_{b} / N_{0}}\right)^{2}\right]
\end{aligned}
$$

For the third error sequence, both $a$ and $b$ approach infinity (the ratio $a / b$, however, approaches unity) as $\delta$ approaches -2. Thus, one must evaluate the pairwise error probability Eq. (15) separately for this case. It is straightforward to show that

$$
\lim _{\substack{a \rightarrow \infty \\ b \rightarrow \infty \\ a / b \rightarrow 1}} f(a, b)=\frac{1}{2} \operatorname{erfc}\left(\sqrt{\frac{b}{2}}-\sqrt{\frac{a}{2}}\right)
$$

Furthermore, in the general case where $\delta \rightarrow-N$, Eq. (21) evaluates to

$$
\lim _{b \rightarrow-N} f(a, b)=\frac{1}{2} \operatorname{erfc}\left(\sqrt{N \frac{E_{b}}{N_{0}}} \cos \phi_{c}\right)
$$

which for $N=2$ and $M=2$ becomes

$$
\lim _{\delta \rightarrow-2} f(a, b)=\frac{1}{2} \operatorname{erfc}\left(\sqrt{\frac{2 E_{b}}{N_{0}}} \cos \phi_{c}\right)
$$


Finally, noting that the Hamming distance $w(\mathbf{u}, \hat{\mathbf{u}})$ is equal to 1 for the first two error sequences and is equal to 2 for the third sequence, substituting Eqs. (23) and (15) combined with Eqs. (19) and (20) into the expression for bit error probability in Eq. (13) gives

$$
P_{b}\left(\phi_{c}\right) \leq f\left(\frac{E_{b}}{N_{0}}\left[\frac{1}{8}\left(\frac{\rho}{E_{b} / N_{0}}\right)^{2}\right], \frac{E_{b}}{N_{0}}\left[2+\left(\frac{\rho}{E_{b} / N_{0}}\right) \cos \phi_{c}+\frac{1}{8}\left(\frac{\rho}{E_{b} / N_{0}}\right)^{2}\right]\right)+\frac{1}{2} \operatorname{erfc}\left(\sqrt{\frac{2 E_{b}}{N_{0}}} \cos \phi_{c}\right)
$$

Finally, the upper bound on average bit error probability $P_{b}$ is obtained by averaging the upper bound in Eq. (24) over the pdf in Eq. (4). Figures 2 and 3 are plots of this upper bound on average probability versus $E_{b} / N_{0}$ in decibels for values of $\rho=7 \mathrm{~dB}$ and $10 \mathrm{~dB}$, respectively. For the purpose of comparison, the exact results (i.e., not an upper bound) for the conventional ideal coherent metric operating in a noisy carrier synchronization environment are

$$
P_{b}=\int_{-\pi}^{\pi} \frac{1}{2} \operatorname{erfc}\left(\sqrt{\frac{E_{b}}{N_{0}}} \cos \phi_{c}\right) p\left(\phi_{c}\right) d \phi_{c}
$$

where $p\left(\phi_{c}\right)$ is given by Eq. (4). Even with only one additional observation symbol interval, considerable savings in $E_{b} / N_{0}$ can be achieved at a fixed error probability, particularly in the region of the knee of the curve where the system begins approaching its irreducible error probability ${ }^{3}$ asymptote (error floor).

\section{Case 2: Binary PSK with $N$-Symbol Observation and Detection $(M=2, N$ arbitrary)}

For $N$ arbitrary, $\delta$ takes on values $-(N-2 i) ; i=0,1,2, \ldots, N-1$. The number of error sequences corresponding to each of these values of $\delta$ is binomially distributed, i.e., there are $\left(\begin{array}{c}N \\ i\end{array}\right)$ sequences that yield a value $\delta=-(N-2 i)$. Furthermore, the Hamming weight associated with each of the $\left(\begin{array}{c}N \\ i\end{array}\right)$ sequences that yield a value $\delta=-(N-2 i)$ is $w(\mathbf{u}, \hat{\mathbf{u}})=N-i$. Finally then, using the above in Eqs. (19) and (22) and substituting the results in Eq. (13), the conditional bit error probability is upper bounded by

$$
\begin{aligned}
P_{b}\left(\phi_{c}\right) & \leq \frac{1}{N}\left[N \frac{1}{2} \operatorname{erfc}\left(\sqrt{N \frac{E_{b}}{N_{0}}} \cos \phi_{c}\right)+\sum_{i=1}^{N-1}\left(\begin{array}{l}
N \\
i
\end{array}\right)(N-i) f\left(a_{i}, b_{i}\right)\right] \\
& =\frac{1}{2} \operatorname{erfc}\left(\sqrt{N \frac{E_{b}}{N_{0}}} \cos \phi_{c}\right)+\sum_{i=1}^{N-1}\left(\begin{array}{c}
N-1 \\
i
\end{array}\right) f\left(a_{i}, b_{i}\right)
\end{aligned}
$$

where

$$
\left\{\begin{array}{l}
b_{i} \\
a_{i}
\end{array}\right\}=\frac{E_{b}}{2 N_{0}}\left\{\left[N+\left(\frac{\rho}{E_{b} / N_{0}}\right) \cos \phi_{c}+\frac{1}{4 i}\left(\frac{\rho}{2 E_{b} / N_{0}}\right)^{2}\right] \pm\left[2 \sqrt{i(N-i)}+\sqrt{\frac{N-i}{i}}\left(\frac{\rho}{E_{b} / N_{0}}\right) \cos \phi_{c}\right]\right\}
$$

\footnotetext{
${ }^{3}$ It is well known [1] that conventional PSK systems exhibit an irreducible error probability (i.e., a finite error probability in the limit as $E_{b} / N_{0}$ approaches infinity) when a noisy carrier synchronization reference with fixed power is used as a demodulation signal. This is observed by examining a curve of $P_{b}$ versus $E_{b} / N_{0}$ with loop SNR, $\rho$, held fixed. The value of this irreducible error probability is given by [1] $\left.P_{b}\right|_{i r r}=\int_{\rho / 2}^{\rho} p\left(\phi_{c}\right) d \phi_{c}$. Note that in practice, as the observation length increases, one should decrease the loop bandwidth of the phase-locked loop (PLL), which results in an increase in the loop SNR, Also, as the bit SNR increases, the loop SNR (for fixed modulation index) increases and thus the error floor decreases.
} 
The upper bound on unconditional average bit error probability is now obtained by averaging Eq. (26) over the pdf in Eq. (4). The numerical results are illustrated in Figs. 2 and 3 for values of $N=4,6$, and 8 . As $N$ gets large, the curves appear to approach an asymptote. This asymptotic behavior is analytically evaluated as follows:

For large $N$, the first term in Eq. (26) when integrated over the pdf in Eq. (4) approaches the irreducible error probability $\left.P_{b}\right|_{i r r}=\int_{\pi / 2}^{\pi} p\left(\phi_{c}\right) d \phi_{c}$. Also, the dominant term in the summation term of Eq. (26) corresponds to $i=N-1$, i.e., $\delta=N-2$. Thus, for large $N$, the second term of Eq. (26) approaches $f\left(a_{N-1}, b_{N-1}\right)$ where

$$
\left\{\begin{array}{l}
b_{N-1} \\
a_{N-1}
\end{array}\right\} \cong \frac{E_{b}}{2 N_{0}}\{N \pm 2 \sqrt{(N-1)}\}
$$

Since from Eq. (28), $\sqrt{b_{N-1}}>\sqrt{b_{N-1}}-\sqrt{a_{N-1}}$, then using the asymptotic form of Eq. (15) for $a$ and $b$ large (see Appendix $A$ of $[5])$, namely,

$$
f(a, b) \cong \frac{1}{2} \operatorname{erfc}\left(\frac{\sqrt{b}-\sqrt{a}}{2}\right)
$$

The value $f\left(a_{N-1}, b_{N-1}\right)$ is obtained as

$$
f\left(a_{N-1}, b_{N-1}\right) \cong \frac{1}{2} \operatorname{erfc}\left\{\sqrt{\frac{E_{b}}{N_{0}}}\right\}
$$

independent of $\phi_{c}$. Finally then, for large $N$, the asymptotic behavior of the average bit error probability is approximately upper bounded by

$$
P_{b} \leq \frac{1}{2} \operatorname{erfc} \sqrt{\frac{E_{b}}{N_{0}}}+2 \int_{\pi / 2}^{\pi} p\left(\phi_{c}\right) d \phi_{c}
$$

namely, the sum of the bit error probability for ideal coherent detection and the error floor. Equation (31) is in very close agreement with the curves for $N=8$ in Figs. 2 and 3.

\section{Case 3: Quaternary PSK With Two-Symbol Observation and Detection $(M=4, N=2)$}

In this case, $E_{0} / N_{0}=2 E_{b} / N_{0}$. There are now a total of $M^{2}-1=15$ possible error sequences each of length 2 . Of these, only eight produce distinct combinations of $|\delta|$ and $\nu$. These are tabulated below:

\begin{tabular}{lccccc}
\hline $\begin{array}{c}\text { Error } \\
\text { sequence }\end{array}$ & Case & $\phi_{k}-\hat{\phi}_{k}$ & $\phi_{k-1}-\hat{\phi}_{k-1}$ & $|\delta|$ & $\nu$ \\
\hline $1,2,3,4$ & 1 & $\pi, 0,3 \pi / 2, \pi / 2$ & $0, \pi, \pi / 2,3 \pi / 2$ & 0 & 0 \\
5,6 & 2 & $0, \pi / 2$ & $\pi / 2,0$ & $\sqrt{2}$ & $\pi / 4$ \\
7,8 & 3 & $0,3 \pi / 2$ & $3 \pi / 2,0$ & $\sqrt{2}$ & $-\pi / 4$ \\
9,10 & 4 & $\pi / 2, \pi$ & $\pi, \pi / 2$ & $\sqrt{2}$ & $3 \pi / 4$ \\
11,12 & 5 & $\pi, 3 \pi / 2$ & $3 \pi / 2, \pi$ & $\sqrt{2}$ & $-3 \pi / 4$ \\
13 & 6 & $\pi / 2$ & $\pi / 2$ & 2 & $\pi / 2$ \\
14 & 7 & $3 \pi / 2$ & $3 \pi / 2$ & 2 & $-\pi / 2$ \\
15 & 8 & $\pi$ & $\pi$ & 2 & $\pi$ \\
\hline
\end{tabular}


The corresponding values of $a$ and $b$ for each of the first five cases which correspond to $|\delta| \neq 2$ are given as follows:

$$
\begin{aligned}
& \left\{\begin{array}{l}
b \\
a
\end{array}\right\}_{1}=\frac{E_{b}}{N_{0}}\left\{\left[2+\left(\frac{\rho}{2 E_{b} / N_{0}}\right) \cos \phi_{c}+\frac{1}{4}\left(\frac{\rho}{2 E_{b} / N_{0}}\right)^{2}\right] \pm\left[2+\left(\frac{\rho}{2 E_{b} / N_{0}}\right) \cos \phi_{c}\right]\right\} \\
& \left\{\begin{array}{l}
b \\
a
\end{array}\right\}_{2}=\frac{E_{b}}{N_{0}}\left\{\left[2+\left(\frac{\rho}{2 E_{b} / N_{0}}\right) \cos \phi_{c}+\frac{1}{4}\left(\frac{\rho}{2 E_{b} / N_{0}}\right)^{2}\right] \pm\left[\sqrt{2}+\frac{1}{\sqrt{2}}\left(\frac{\rho}{2 E_{b} / N_{0}}\right)\left(\cos \phi_{c}+\sin \phi_{c}\right)\right]\right\} \\
& \left\{\begin{array}{l}
b \\
a
\end{array}\right\}_{3}=\frac{E_{b}}{N_{0}}\left\{\left[2+\left(\frac{\rho}{2 E_{b} / N_{0}}\right) \cos \phi_{c}+\frac{1}{4}\left(\frac{\rho}{2 E_{b} / N_{0}}\right)^{2}\right] \pm\left[\sqrt{2}+\frac{1}{\sqrt{2}}\left(\frac{\rho}{2 E_{b} / N_{0}}\right)\left(\cos \phi_{c}-\sin \phi_{c}\right)\right]\right\} \\
& \left\{\begin{array}{l}
b \\
a
\end{array}\right\}_{4}=\frac{E_{b}}{N_{0}}\left\{\left[2+\left(\frac{\rho}{2 E_{b} / N_{0}}\right) \cos \phi_{c}+\frac{3}{4}\left(\frac{\rho}{2 E_{b} / N_{0}}\right)^{2}\right] \pm\left[\sqrt{2}+\frac{1}{\sqrt{2}}\left(\frac{\rho}{2 E_{b} / N_{0}}\right)\left(3 \cos \phi_{c}+\sin \phi_{c}\right)\right]\right\} \\
& \left\{\begin{array}{l}
b \\
a
\end{array}\right\}_{5}=\frac{E_{b}}{N_{0}}\left\{\left[2+\left(\frac{\rho}{2 E_{b} / N_{0}}\right) \cos \phi_{c}+\frac{3}{4}\left(\frac{\rho}{2 E_{b} / N_{0}}\right)^{2}\right] \pm\left[\sqrt{2}+\frac{1}{\sqrt{2}}\left(\frac{\rho}{2 E_{b} / N_{0}}\right)\left(3 \cos \phi_{c}-\sin \phi_{c}\right)\right]\right\}
\end{aligned}
$$

For cases 6 and 7, the following is analogous to Eq. (22):

$$
\lim _{\delta \rightarrow \pm j N} f(a, b)=\frac{1}{2} \operatorname{erfc}\left(\sqrt{\frac{N E_{b}}{2 N_{0}}}\left(\cos \phi_{c} \pm \sin \phi_{c}\right)\right)
$$

which for $N=2$ and $M=4$ becomes

$$
\lim _{\delta \rightarrow \pm j 2} f(a, b)=\frac{1}{2} \operatorname{erfc}\left(\sqrt{\frac{2 E_{b}}{N_{0}}}\left(\cos \phi_{c} \pm \sin \phi_{c}\right)\right)
$$

Finally, for case 8, Eq. (22) is used to obtain

$$
\lim _{\delta \rightarrow-2} f(a, b)=\frac{1}{2} \operatorname{erfc}\left(\sqrt{\frac{4 E_{b}}{N_{0}}} \cos \phi_{c}\right)
$$

Evaluating the Hamming distances for the 15 error sequences and substituting the above results into the expression for the bit error probability bound in Eq. (13) gives

$$
\begin{aligned}
P_{b}\left(\phi_{c}\right) \leq & \frac{1}{4}\left\{6 f\left(a_{1}, b_{1}\right)+2 f\left(a_{2}, b_{2}\right)+2 f\left(a_{3}, b_{3}\right)+4 f\left(a_{4}, b_{4}\right)+4 f\left(a_{5}, b_{3}\right)\right\} \\
& +\frac{1}{4}\left\{\operatorname{erfc}\left(\sqrt{\frac{2 E_{b}}{N_{0}}}\left(\cos \phi_{c}+\sin \phi_{c}\right)\right)+\operatorname{erfc}\left(\sqrt{\frac{2 E_{b}}{N_{0}}}\left(\cos \phi_{c}-\sin \phi_{c}\right)\right)+\operatorname{erfc}\left(\sqrt{\frac{4 E_{b}}{N_{0}}} \cos \phi_{c}\right)\right\}
\end{aligned}
$$


Figures 4 and 5 are comparable to Figs. 2 and 3 for the $M=4$ (QPSK) case. The analytical exact result corresponding to the ideal coherent metric operating in a noisy carrier synchronization environment is now [1]

$$
P_{b}=\int_{-\pi}^{\pi} \frac{1}{4} \operatorname{erfc}\left(\sqrt{\frac{E_{b}}{N_{0}}}\left(\cos \phi_{c}+\sin \phi_{c}\right)\right) p\left(\phi_{c}\right) d \phi_{c}+\int_{-\pi}^{\pi} \frac{1}{4} \operatorname{erfc}\left(\sqrt{\frac{E_{b}}{N_{0}}}\left(\cos \phi_{c}-\sin \phi_{c}\right)\right) p\left(\phi_{c}\right) d \phi_{c}
$$

Analysis and plots for larger values of $N$ are not included here, but they would show further improvement as was true for the binary case.

\section{E. Performance Sensitivity to Mismatch In Loop SNR}

Here the authors investigate the sensitivity of the average bit error probability (in terms of its upper bound) of the MLSE receiver to a mismatch between the true loop SNR, $\rho$, and the estimate of it, $\hat{\rho}$, supplied to the implementation of Fig. 1. In particular, the authors evaluate, for the special cases of Sections III.B and III.C, the upper bound

$$
P_{b} \leq \int_{-\pi}^{\pi} P_{b u}\left(\phi_{c} ; \hat{\rho}\right) p\left(\phi_{c}\right) d \phi_{c}
$$

where $P_{b u}\left(\phi_{c} ; \hat{\rho}\right)$ is given by the upper bound in Eq. (24) or Eq. (26) with $\rho$ replaced by $\hat{\rho}=\rho[1+(\hat{\rho}-\rho) / \rho] \triangleq$ $\rho(1+\varepsilon)$ and $p\left(\phi_{c}\right)$ is as given by Eq. (4). Figures 6 and 7 are illustrations of Eq. (38) for $M=2, \rho=10 \mathrm{~dB}$, and $N=2$ and 8 , respectively, with fractional mismatch $\epsilon$ as a parameter. One observes that even with mismatches as much as 50 percent $(\epsilon= \pm 0.5)$, there is negligible effect on the error probability performance. Thus, the authors conclude that the MLSE receiver is quite insensitive to mismatch in the loop SNR.

\section{Conclusions}

By making use of the known (or estimated) value of loop SNR in the decision metric, it is possible to improve the error probability performance of a partially coherent MPSK system relative to that corresponding to the commonly used ideal coherent decision rule. Using a maximum-likelihood approach, an optimum decision metric was derived and shown to take the form of a weighted sum of the ideal coherent decision metric (i.e., correlation) and the noncoherent decision metric previously shown to be optimum for differential detection of MPSK. The performance of a receiver based on this optimum decision rule improves with the increasing length of the observation interval (data symbol sequence length). Furthermore, the performance is quite insensitive to mismatch between the estimate of loop SNR (e.g., obtained from measurement) fed to the decision metric relative and its true value. 


\section{References}

[1] W. C. Lindsey and M. K. Simon, Telecommunication Systems Engineering, New York: Prentice-Hall, Inc., 1973.

[2] D. Divsalar and M. K. Simon, "Multiple-Symbol Differential Detection of MPSK," IEEE Transactions on Communications, vol. 38, no. 3, pp. 30-308, March 1990.

[3] S. Stein, "Unified Analysis of Certain Coherent and Noncoherent Binary Communications Systems," IEEE Transactions on Information Theory, vol, IT-10, pp. 43-51, January 1964.

[4] J. Marcum, Tables of $Q$ Functions, RAND Corporation Report M-339, Santa Monica, California: Rand Corporation, January 1950.

[5] M. Schwartz, W. R. Bennett, and S. Stein, Communication Systems and Techniques, New York: McGraw-Hill, Inc., 1966. 


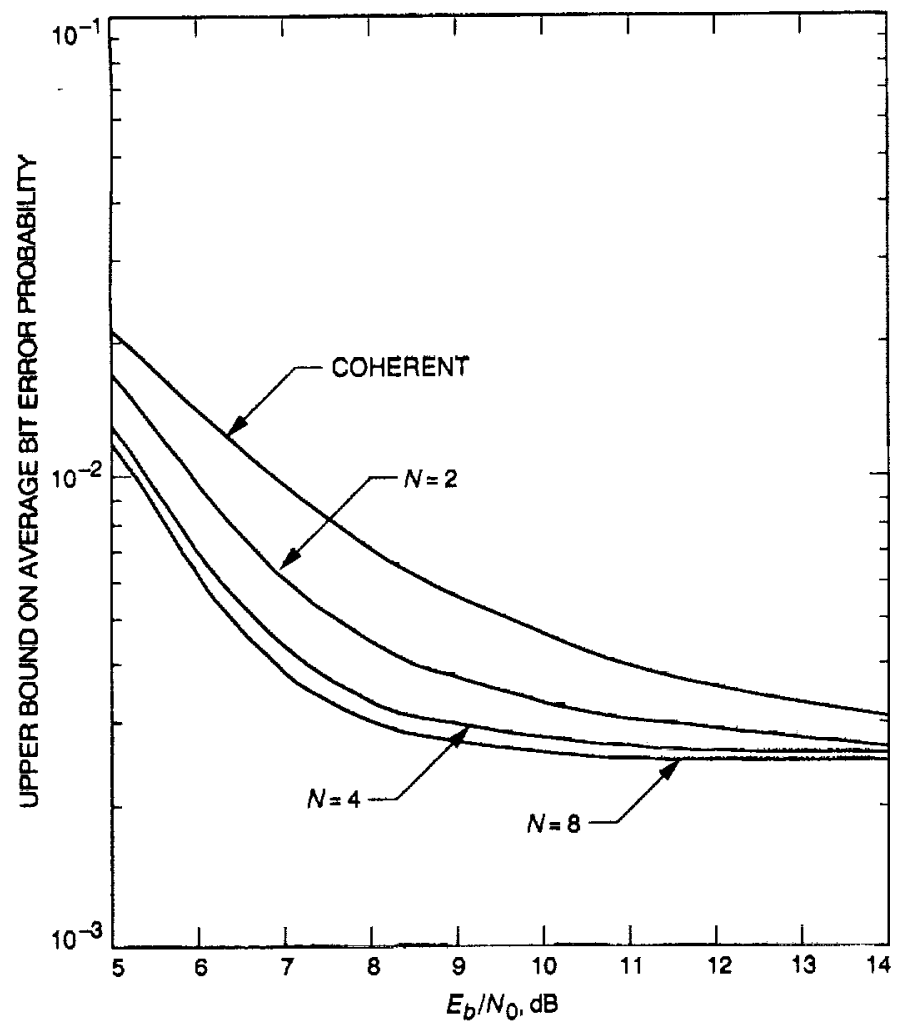

Flg. 2. Upper bound on average blt error probabillity versus $E_{b} / N_{0}$ in declbels for MLSE with $N$ as a parameler; $M=2$ and $\rho=7 \mathrm{~dB}$.

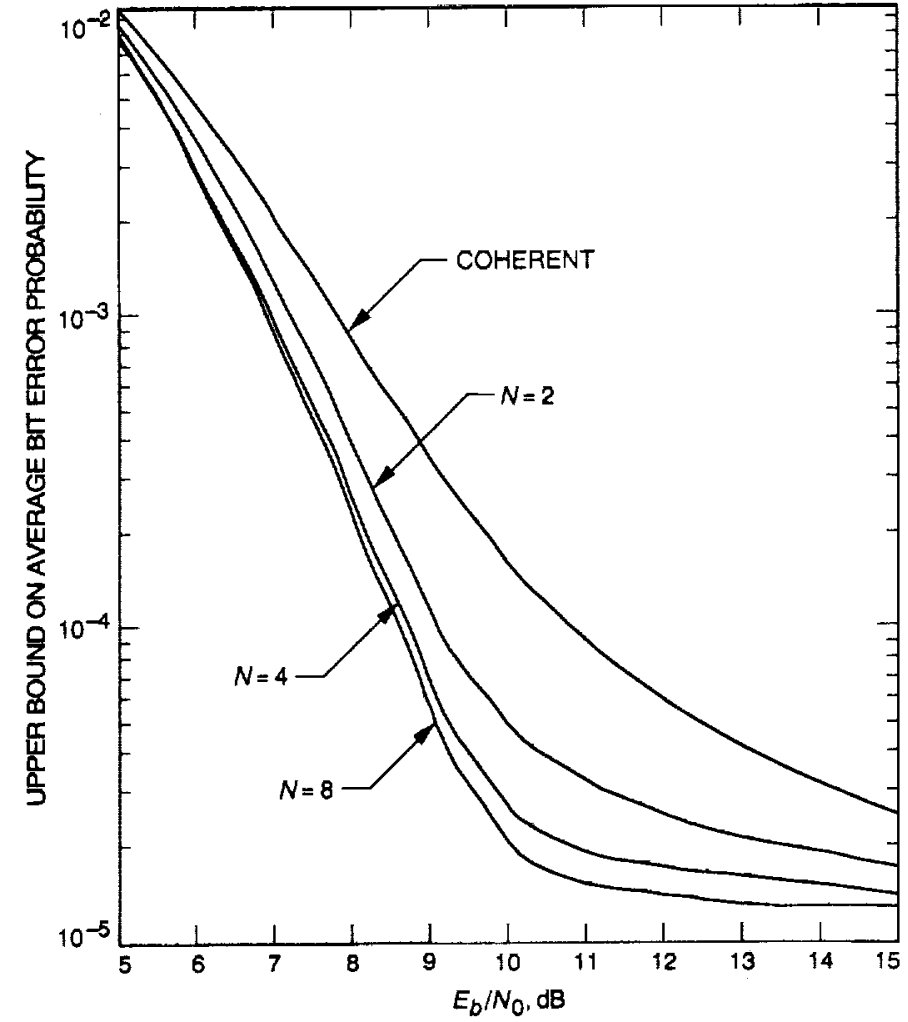

Fig. 3. Upper bound on average blt error probabillty versus $E_{b} / N_{0}$ In declbels for MLSE with $N$ as a parameter; $M=2$ and $\rho=10 \mathrm{~dB}$. 


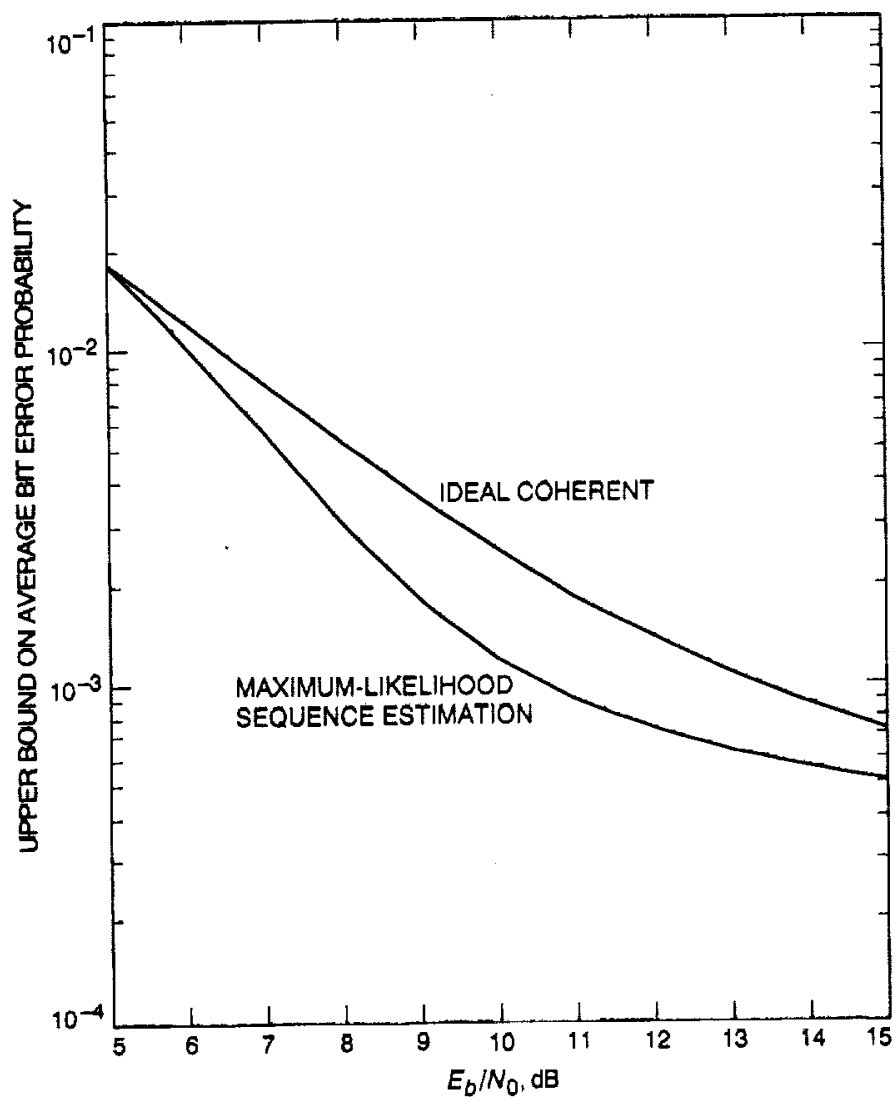

Flg. 4. Upper bound on average bit error probabllity versus $E_{b} / N_{0}$ in declbels for MLSE and comparlson with exact performance of ideal coherent metric; $M=4, N=2$, and $\rho=13 \mathrm{~dB}$.

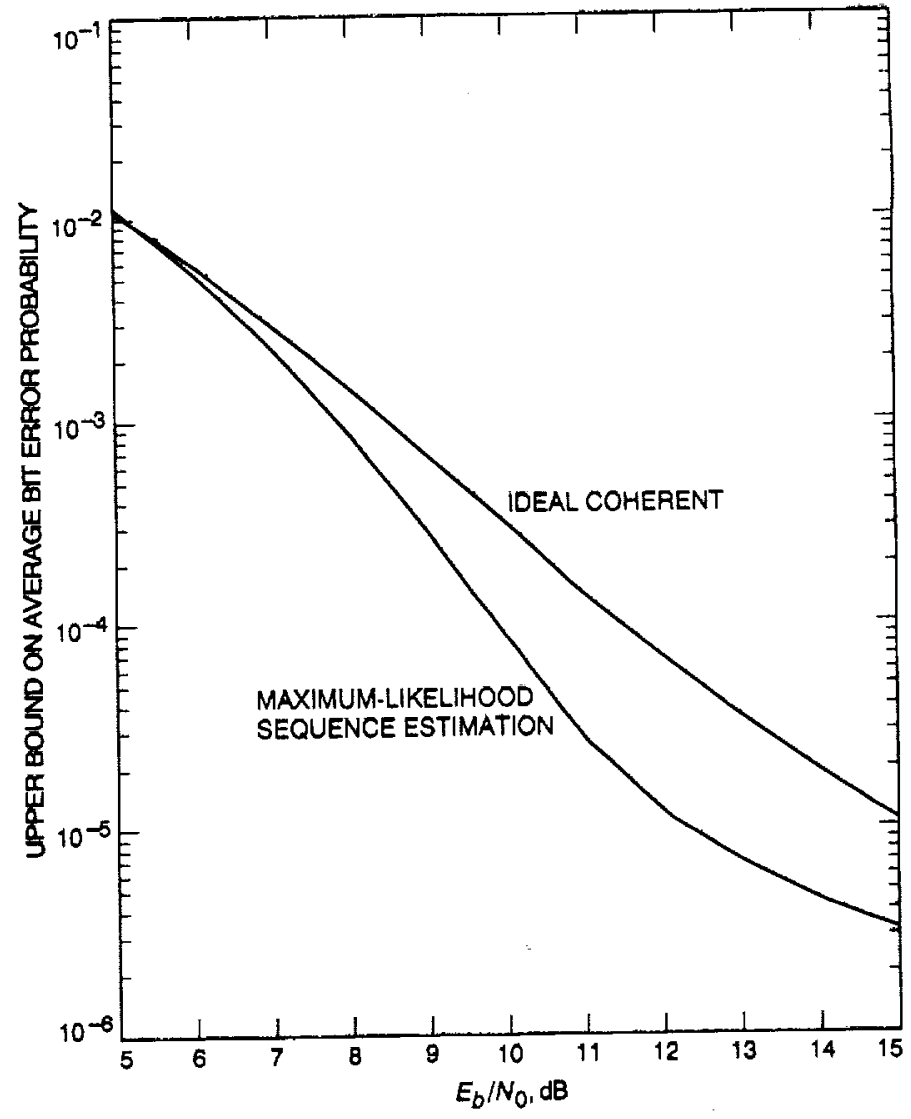

Fig. 5. Upper bound on average blt error probabllity versus $E_{b} / N_{0}$ In decibels for MLSE and comparison with exact performance of ideal coherent metric; $M=4, N=2$, and $\rho=16 \mathrm{~dB}$. 


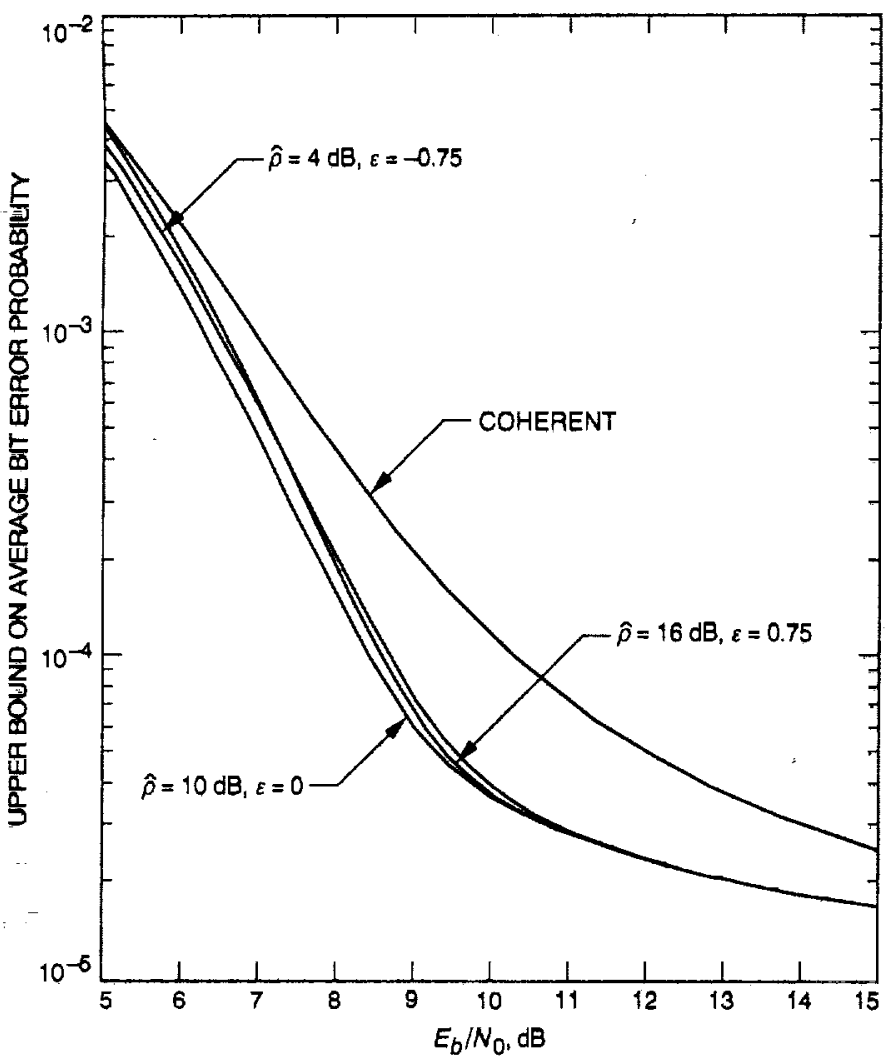

Flg. 6. Upper bound on average blt error probabllity versus $E_{b} / N_{0}$ in decibels for MLSE in the presence of loop SNR mismatch; $M=2$, $N=2$, and $\rho=10$.

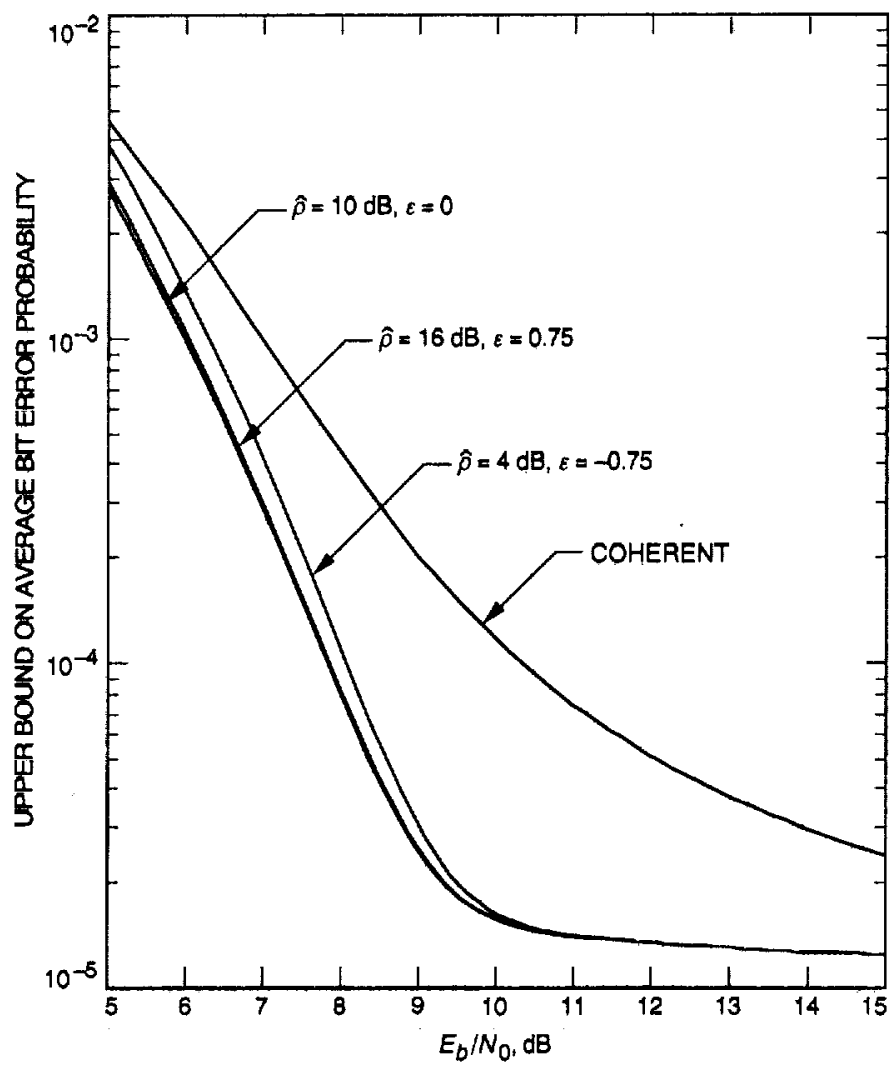

Fig. 7. Upper bound on average blt error probabllity versus $E_{b} / N_{0}$ In declbels for MLSE in the presence of loop SNR miematch; $M=2$, $N=8$, and $\rho=10$. 